\title{
FRICTION AND WEAR PROCESSES IN TRIBOTECHNICAL SYSTEM
}

\author{
Karimov Sh.A. ${ }^{1}$, Mamirov Sh.Sh. ${ }^{2}$, Khabibullayeva I.A. ${ }^{1}$, Bektemirov B.Sh. ${ }^{1}$, Khusanov N.1 \\ ${ }_{1}^{1}$ Materials Science department, Tashkent State Technical University, Uzbekistan \\ 2Tashkent State Technical Unversity Almalyk branch, Uzbekistan \\ Email: begalibektemirov94@gmail.com
}

\begin{abstract}
Friction and wear processes take place in the surface layer, which plays a special role in the tribotechnical system. Plastic deformation of the surface layer during friction causes specific structural changes (fragmentation of the structure) and the development of stress concentrators of different scales (bifurcation centers). With structural self-organization, bifurcation centers are transformed either into dissipative sources and generators of various carriers of plastic deformation or into centers of material destruction.
\end{abstract}

Keywords: Friction, wear, coatings, wear resistance, stress, heat, diffusion, secondary structures.

\section{Introduction}

The development of a unified theory of the tribotechnical process is faced with the difficulty of a wide variety of friction and wear mechanisms, which are a complex combination of physicochemical and mechanical processes occurring at different scales of contact interaction, not limited to three main levels: micro, meso, and macro.

Thus, microstructural dynamics, changes in the chemical composition of the material, generation of a flow of crystal structure defects and wear products occur at scale levels of the order of $10 \ldots 100 \mathrm{~nm}$, and topographic changes in the friction surface that determine the density of the flow of mechanical energy are formed at levels of the order of $0.1 \ldots 1.0$ mm ... The difference between these levels by 9 orders of magnitude makes it impossible to describe them within the framework of a single model. Physical mesomechanics does not solve this problem but reveals the deformation relationship between the macro, meso, and micro levels, through which energy flows are transferred and dissipated in the contact process of friction and wear.

\section{Materials and Methods}

Recall that physical mesomechanics is a science that studies changes in the structure and properties of a solid during plastic deformation based on the fundamental laws of thermodynamics of nonequilibrium processes, physics, and chemistry of condensed matter. The task of physical mesomechanics concerning wear processes in friction pairs is to reveal the patterns of structural changes in the surface layers, leading to the development of fragmented mesostructure formations, from which wear products are subsequently generated. The sequence and nature of these processes are initiated by the flow of mechanical energy of contact interaction, the main form of relaxation of which is plastic deformation [13].

Surfaces and adjacent layers are of particular importance in the case of deformation and destruction of the material, therefore they stand out as a separate structural system, and it is assigned the main role in the processes of surface stress concentrators functioning, stress relaxation, and dissipation of supplied energy. During friction and wear, the role of surface layers increases significantly because through them there is a transfer of mechanical energy of contact interaction, and all processes of a tribological nature are localized in them. A juvenile (freshly formed) surface, in essence, is a macroscopic defect in the crystal structure, consisting of atoms with broken and unbalanced chemical bonds, which in aggregate manifest themselves as surface energy.

The presence of stress concentrators of micro and mesogeometric nature is determined by the very structure of the surface, which cannot be ideally smooth. Due to the crystallographic misorientation of crystallites relative to the surface, it is covered with kinks, protrusions, vacancies, and adsorbed atoms. Kinks and protrusions of the microscale level are thermodynamically equilibrium, the concentration of which can be determined by the equation [4].

$$
N=2 r_{0} \cdot \exp \left(-\frac{E_{u}}{k T}\right)
$$

where $r_{0}$ is the distance between atoms in the direction of the step; $E_{k}$ is the energy required to form a kink. 
Surface layer thermodynamics. The laws of thermodynamics are universal; therefore, their application makes it possible to identify and generalize the variety of relationships between properties and factors that determine these properties. The surface layer as a thermodynamic system is multiphase and inhomogeneous. Plastic deformation of the surface is always accompanied by an increase in its area, which can be represented as a process of the release of crystal atoms from the bulk to the surface. In this case, to maintain a constant area of actual contact during ongoing plastic deformation, rotation of atoms emerging from the volume to the surface and entering from the surface into the volume is required. Diffusion processes are unable to provide high-speed rotation of atoms, although the diffusion rate in the surface layers reaches anomalously high values, and this is achieved due to rotational fragmented mesostructures.

The thermodynamic equation of the deformation process in the surface layers during friction can be described by the general expression (2), but taking into account the active role played by surfaces, including the internal interfaces, it is necessary to introduce an additional term,

$$
T \cdot d S=d E+P \cdot d V-\sum_{i=1}^{n} \mu_{i} \cdot d C_{i}-\sum_{j=1}^{m} \sigma_{A j} \cdot d A_{j}
$$

where $\sigma_{A}$ - specific surface tension; A is the surface area; $m$ is the number of surface types.

Specific surface tension $\left(\sigma_{\mathrm{A}}\right)$ is a more general character than specific surface energy $(\gamma)$, and they are related by the dependence

$$
\sigma_{A}=\gamma+A \cdot \frac{\partial \gamma}{\partial A}
$$

On smooth, and in the general case, non-fractal surfaces, and at $\gamma$ - const, the specific surface tension is numerically equal to the specific surface energy.

For open surfaces, we can assume with some approximation that $\sigma_{\mathrm{A}}=\gamma$. But at the inner boundaries of the division, the equality between these values is impracticable. Depending on the degree of surface development, the second term in Eq. (3) can take different values, both in magnitude and in sign. With an increase in the degree of development of the boundaries, the specific surface tension increases, making the boundary less mobile.

The last two terms on the right-hand side of equation (2) represent the energy consumption for the processes of formation of secondary structures, deformation fragmentation of the mesostructure, and change in the shape of the friction surface, leading to the disclosure of new surfaces. The activity of these processes largely depends on the power density of the dissipative sources. The wear resistance of the structure is ensured by the highest activity of the dissipative channels.

The processes of setting and the formation of adhesive and cohesive bonds occur only in those microlocal zones where contact loads develop that exceed the strength of the boundary layers, and zones of stress concentrators of rubbing surfaces are exposed. The dynamics of the development of zones of adhesion-cohesion interaction are wandering, depending on the reference curve of microroughness, and the frequency of formation is stochastic. The formation of adhesive bonds occurs due to normal contact loads, and destruction due to tangential stresses caused by sliding. The microlocal zone of the adhesive process is subjected to compression during the formation of an adhesive bond and shear deformation during their destruction.

Consequently, in the plastic deformation of the tribo process, there can be no tensile components perpendicular to the plane of friction, and this casts doubt on many models of mechanical abrasion, in which the activity of generation of wear products is associated with the separation of particles and the ultimate tensile strength of the material [5-8].

\section{- The peculiarity of deformation processes during friction}

A high concentration of plastic deformation in the surface layers leads to the development of stress fields, deformations, and displacement rates with very high gradient characteristics. This, in turn, causes loosening and delamination of the material at the mesoscale level along the shear rate rupture planes, and the formation of interfaces parallel to the friction plane. Each layer initially consists of dendrites oriented parallel to the slip plane, and their degree of order is of a gradient nature, the vector of which is directed to the slip plane. The formation of such boundaries is one of the forms of self-organization of the mesostructure of a tribological nature.

The main carriers of deformation of contact surfaces during friction, in addition to traditional dislocations, disclinations, and grain boundary shears, are displacements between layers separated by interfaces parallel to the friction plane, as well as rotational displacements at the sites of rupture of adhesive bonds due to tangential stresses.

As the tribological process develops further, the dendritic structure breaks down and transforms into an ultradispersed polycrystalline structure. In fig. 1 shows the surface microstructure, where shear planes and delamination regions are visible, in which the process of formation of ultradispersed grains began. In the layers adjacent to the friction surface, grain refinement occurs more actively, and fragments of the micro-and mesostructure are visible in them, from which wear particles can subsequently arise. 
The high diffusion and chemical activity of an ultradispersed structure lead to its complex interaction with the environment, as a result of which a structure is formed that is very different in chemical composition from the original one. The layer, consisting of an ultradispersed structure, has a relatively uniform profile of the distribution of the chemical potential over the thickness and a sharp interface with the substrate.

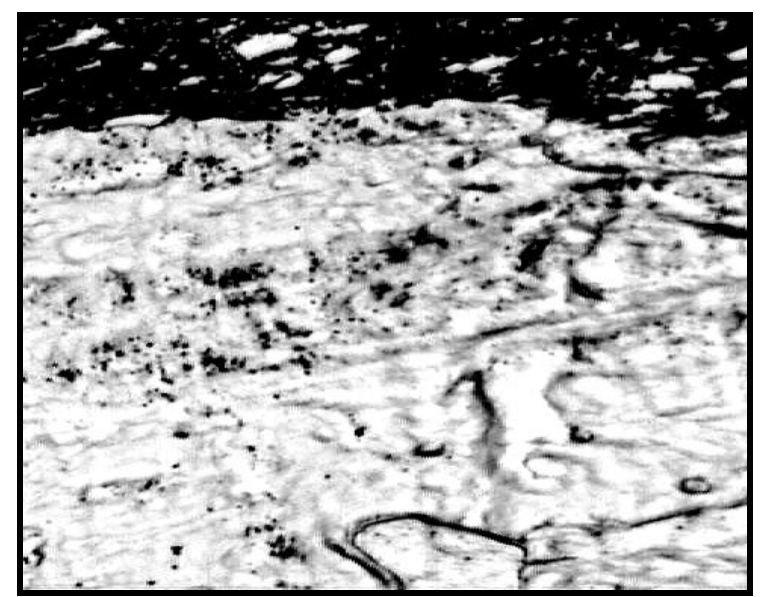

Figure 1: Breakdown of the dendritic microstructure in the surface layers of a block made of steel 3 during friction against carbon steel $U 10$ (x1000)

The interfaces of the ultradispersed structure are highly developed. Considering that any interfaces have higher mobility in comparison with other structural components, localization of carriers of plastic deformation and sources of dissipation of the mesoscale level occurs in them. This means that with an increase in the development of grain and phase boundaries, the wear resistance of the material should increase.

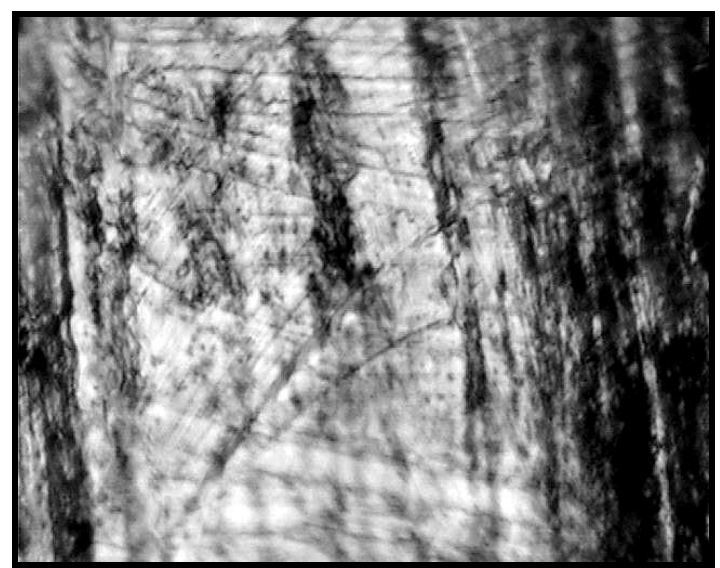

Figure 2: Microcracks perpendicular to the direction of the sliding velocity vector on the friction surface of the copper disk (x1000)

The wear rate is determined by the ratio of the activities of these two processes.

\section{Research results and Discussion}

Microcracks that appear in front of the seizure zone are oriented mainly at an angle of $\pi / 4$ to the sliding velocity vector since the cause of their appearance is the compression of the surface layers. Examples of such microcracks are shown in Fig. 2 and 3.

The location of microcracks is ordered, and they alternate with groove stripes similar to the neck of localized tensile deformation. Microcracks consist of short "banks", and localized bands of tensile deformations penetrate the entire "path" of sliding.

The fields of compressive and tensile stresses on the friction surface are periodic, forming a wave relief of the "checkerboard" type on the surface, therefore, the microcracks distributed on the surface form a mesh pattern of superimposed orthogonal and oblique (at an angle of $\pi / 4$ ) relative to the sliding velocity vector marks (Fig. 4), reminiscent of the tread of a car tire.

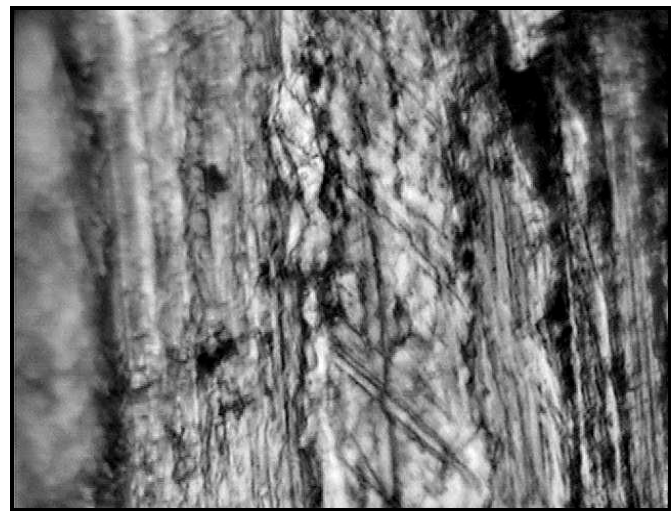

Figure 3: Microcracks at an angle of $\pi / 4$ to the direction of the sliding velocity vector on the friction surface of a copper disk (x1000)

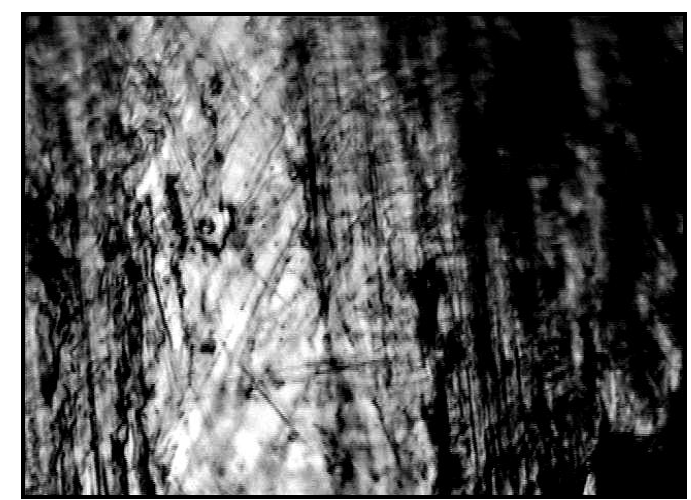

Figure 4: Microcracks on the friction surface of a copper pad (x1000)

In fig. 5 shows the friction surface, on which traces of displacement and "creeping" of the layers are visible, and Fig. 6 shows the microstructure of the subsurface layer, where zones of displacement and formation of micropores and cracks are visible. 
The friction surface covered with microcracks is an independent dissipative structure of a tribological nature, which can play an active role in the dissipation of mechanical energy.

The process of mechanical abrasion is the final stage of most wear mechanisms, and the formation of wear products occurs mainly due to rotational displacements of the material in the zones of rupture of sliding velocities. Let us consider this process in an idealized form with friction without lubricant. Initially, plastic deformation is localized in the area adjacent to the friction surface. Then, the plastic flow intensively develops in the near-surface layer, causing mesofragmentation of the material and the development of a turbulent layer.

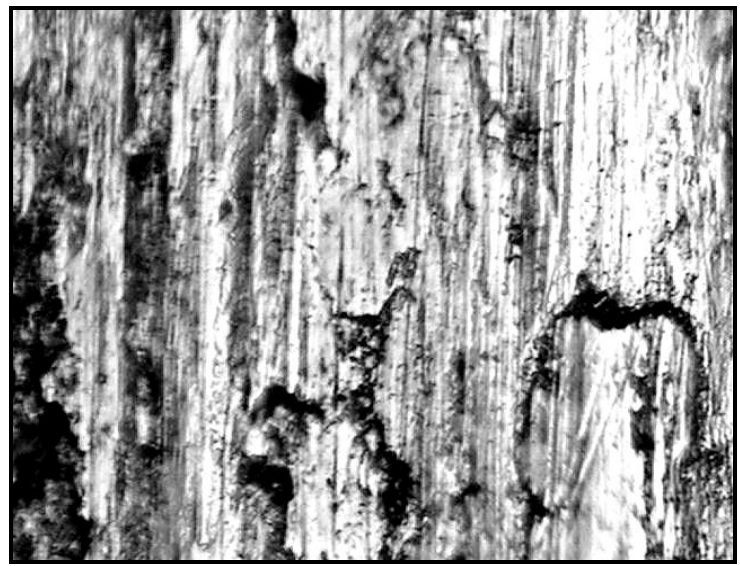

Figure 5: «Crawling» Creep of a fragmented layer on the friction surface of a copper disk ( $x 400)$

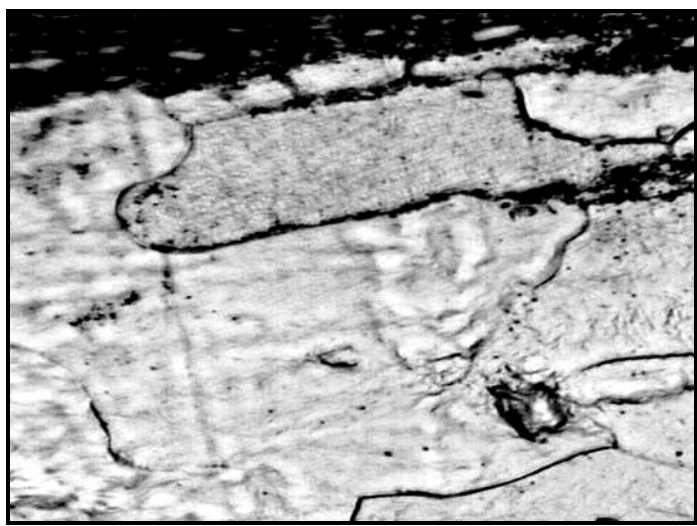

Figure 6: Microstructure of the subsurface layer of a shoe made of steel 3 after friction against steel U1O (x1000)

The formation of elements of a turbulent layer represents the final stage of a single mesofragmentation process and goes through several stages of its development.

The formation of microcracks in the adhesive contact zone and a high degree of fragmentation of the surface layer structure bring the material in this localized region into a complex-stressed state, in which rotational modes of the strain tensor are most actively manifested.
The development of rotary modes occurs due to the moments from the tangential seizing forces, oriented parallel to the sliding direction, and the stress gradient of internal friction in the surface layers. This process is shown schematically in Fig. 7, where the seizure zones, the sliding direction $\left(\mathrm{V}_{\mathrm{sl}}\right)$, and the direction of the rotary modes are marked.

As a result, the crack located on the left in the diagram opens up, bringing a new surface into the friction zone, and the crack on the right contracts and carries a part of the friction surface into the depth of the material. Thus, the formation of a fragmented pivot element and atomic rotation occurs.

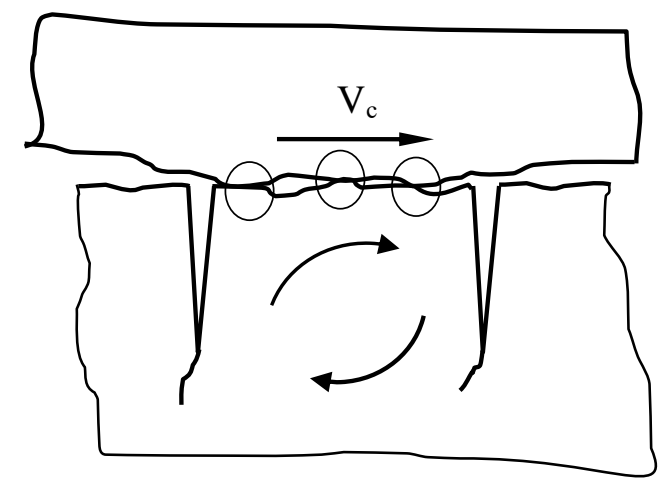

Figure 7: A diagram of the formation of a turbulent fragment (roller)

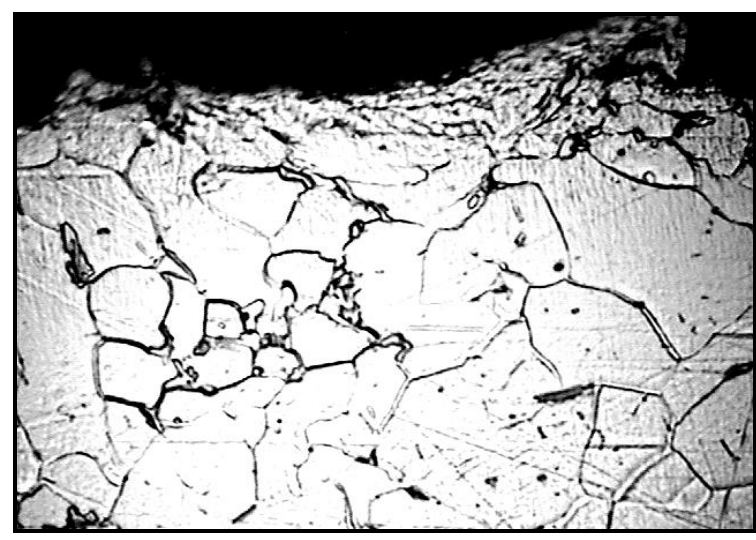

Figure 8: The friction surface of a specimen made of steel 20 at the moment of formation of a "bead"

(x1000)

The steady-state wear process consists of the stable functioning of all mechanisms of structure formation, fragmentation, the development of a turbulent layer, and the formation of wear particles, which together can be considered as a sequence of alternating homeostasis of the tribological system.

Strain hardening during friction forms a positive gradient of mechanical properties in the surface layers due to the development of residual compressive internal stresses, which stabilize the growth of microcracks and increase their stability. 


\section{Conclusions}

Plastic deformation during friction is localized in the surface layer and is accompanied by fragmentation of the structure, the development of stress concentrators of different scale levels, which play the role of bifurcation centers, transforming either into dissipative sources or into centers of material destruction.

Self-organization of the development of secondary structures during friction is manifested in ordered fragmentation, the formation of microcracks oriented in a certain direction relative to the sliding velocity vector, and active sources of dissipation that generate heat release and the flow of plastic deformation carriers.

The formation of wear products occurs due to rotational displacements of the mesolayers of the material in the zones of discontinuity of sliding velocities, while the wear process itself can be considered as an active dissipative process that removes the energy flow from the friction zone due to the flow of matter.

\section{References}

[1] Spravochnik po tribonike [Tribonics Handbook] /Pod obsch. ed. M. Hebdy, A.V. Chichinadze. T.1. Teoreticheskie osnovy [Theoretical basis]. - M.: Mashinostroenie, P: 400, 1989.
[2] Panin V.E., Kolubaev A.V., Slosman A.I., Tarasov S.Y., Panin S.V., Sharkeev Y.P.; Iznos v parah treniya kak zadacha fizicheskoi mezomehaniki [Wear in friction pairs as a problem of physical mesomechanics], Fizicheskaya mezomehanika, Volume 1, Pp: 67-74, 2000.

[3] Bolohonov R.R., Bolesta A.V., Panin V.E. et al.; Poverhnostnye sloi i vnutrennie granicy razdela $\mathrm{V}$ geterogennyh materialah [Surface layers and internal interfaces in heterogeneous materials], under ed. V.E. Panin, Novosibirsk: pub SO RAN, 500p, 2006.

[4] Sulima A.M., Shulov V.A., Yagodkin Y.D.; Poverhnostnyi sloi i ekspluatacionnye svoistva detalei mashin [Surface layer and performance properties of machine parts], Mashinostroenie, 240p, 1988.

[5] Erkin, U., Umidjon, M., \& Umida, S. (2021, September). Application of Magnetic Field on Lubricating Cooling Technological Condition in Metal Cutting Process. In International Conference on Reliable Systems Engineering (pp. 100-106). Springer, Cham.

[6] Mur D.; Osnovy i primenenie triboniki [Basics and Applications of Tribonics], Mir, 488p, 1978.

[7] Makushok E.M., Kalinovskaya T.V., Beliy A.V.; Massoperenos $\mathrm{v}$ processah treniya [Mass transfer in friction processes], Minsk, Nauka i tehnika, 272p, 1978.

[8] Chixos X.; Sistemnyi analiz v tribonike [System analysis in tribonics], pub: Mir, 344p, 1982. 